\title{
Fate mapping of gallbladder progenitors in posteroventral foregut endoderm of mouse early somite-stage embryos
}

\author{
Mami UEMURA ${ }^{1)}$, Hitomi IGARASHI ${ }^{1}$, Aisa OZAWA ${ }^{1)}$, Naoki TSUNEKAWA ${ }^{1)}$, Masamichi KUROHMARU ${ }^{1)}$, \\ Masami KANAI-AZUMA ${ }^{2)}$ and Yoshiakira KANAI ${ }^{1) *}$ \\ 1) Department of Veterinary Anatomy, The University of Tokyo, 1-1-1 Yayoi, Bunkyo-ku, Tokyo, 113-8657, Japan \\ ${ }^{2)}$ Center for Experimental Animals, Tokyo Medical and Dental University, 1-5-45 Yushima, Bunkyo-ku, Tokyo 113-8510, Japan
}

(Received 2 December 2014/Accepted 22 December 2014/Published online in J-STAGE 7 January 2015)

\begin{abstract}
In early embryogenesis, the posteroventral foregut endoderm gives rise to the budding endodermal organs including the liver, ventral pancreas and gallbladder during early somitogenesis. Despite the detailed fate maps of the liver and pancreatic progenitors in the mouse foregut endoderm, the exact location of the gallbladder progenitors remains unclear. In this study, we performed a DiI fate-mapping analysis using whole-embryo cultures of mouse early somite-stage embryos. Here, we show that the majority of gallbladder progenitors in 9-11-somite-stage embryos are located in the lateral-most domain of the foregut endoderm at the first intersomite junction level along the anteroposterior axis. This definition of their location highlights a novel entry point to understanding of the molecular mechanisms of initial specification of the gallbladder.
\end{abstract}

KEY WORDS: fate mapping, foregut, gallbladder, mouse

doi: 10.1292/jvms.14-0635; J. Vet. Med. Sci. 77(5): 587-591, 2015

In mouse early embryogenesis, the foregut endoderm first arises at the early to mid-streak stages, leading to the formation of one endodermal sheet layer. The foregut endoderm sheet covers the anterior surface of the embryonic region by the early head fold stage and then undergoes the formation of foregut invagination, the so-called anterior intestinal portal (AIP), during early somitogenesis [10, 27, 28]. During AIP formation and subsequent foregut tubulogenesis, a medial part of the foregut endoderm sheet is roughly defined in the dorsal walls of the primitive gut tubes, and the lateral foregut endoderm cells on both the left and right sides are relocated into the ventral midline of the gut tube, leading to gut tube closure and embryonic turning. During foregut closure, the liver, gallbladder and ventral pancreas primordia are separately specified within the adjacent close regions of the ventral foregut endoderm, and the formation of each primordium could be induced and maintained by distinct signaling factors, such as FGFs, BMPs or TGF $\beta$, emitted from the cardiac mesoderm or transverse septum [2, 16, 27].

Sry-related HMG box gene-17 (Sox17) [7] is well known as a master regulatory gene for initial endoderm specification $[6,15,19,22]$. Sox 17 is transiently activated in the foregut endoderm during the early primitive stage $(7.0 \mathrm{dpc})$ to early headfold stage $(7.5 \mathrm{dpc})[6,18,25]$, and its expression is rapidly reduced by the late headfold stage. Interestingly, Sox 17 appeared to be reactivated in a small area of the fore-

*Correspondence to: Kanai, Y., Department of Veterinary Anatomy, The University of Tokyo, 1-1-1 Yayoi Bunkyo-ku, Tokyo 113-8657, Japan. e-mail: aykanai@mail.ecc.u-tokyo.ac.jp

(C)2015 The Japanese Society of Veterinary Science

This is an open-access article distributed under the terms of the Creative Commons Attribution Non-Commercial No Derivatives (by-nc-nd) License $<$ http://creativecommons.org/licenses/by-nc-nd/3.0/>. gut endoderm, including the gallbladder primordium, during early somitogenesis, and its high expression is continuously maintained within the distal edge of the gallbladder primordium at least through the late organogenic stages $(\sim 15.5 \mathrm{dpc})$ $[11,21,25]$. Moreover, loss-of-function and gain-of-function analyses of the Sox 17 gene in the early organogenic stages $(9.5-10.5 \mathrm{dpc})$ demonstrated that it is essential for the formation of gallbladder primordia in a cell-autonomous fashion $[21,25]$. Full Sox 17 activity is also required for the development and maintenance of the gallbladder and cystic duct at the perinatal stages $(16.5-18.5 \mathrm{dpc})$, because haploinsufficiency of the murine Sox 17 gene leads to severe gallbladder hypoplasia, frequently accompanied by bile duct stenosis/ atresia at birth [26]. These findings, therefore, suggest that Sox 17 activity is required both for the initial formation and for the maturation and maintenance of the gallbladder and cystic duct in a dose-dependent fashion.

During the 9-11 somite stages (8.5-8.75 dpc), Sox 17 is up-regulated in a small defined area of the lateral foregut endoderm, which is located posterolaterally to the AIP lip at the boundary between the first and second somites (around the first intersomite junction) along the anteroposterior axis (see inset in Fig. 1A), and its upregulation occurs several hours after the onset of Hex expression in the liver buds (at the 8-9-somite stages) and at the onset of $P d x 1$ upregulation in the pancreatic region (in the midline of the AIP lip at the 10-11-somite stages; [25]). This suggests that the small Sox 17-positive region is a potential site for the initial specification of gallbladder progenitors in foregut endoderm cells at the 10-11 somite stages. However, during the early somite stages (8.25-8.5 dpc), the hematopoietic and vascular endothelial cells also begin to express Sox 17 in the mesodermal region underneath the ventral foregut endoderm and the AIP lip $[3,8,14,17]$. Moreover, a portion of the ventral pancre- 

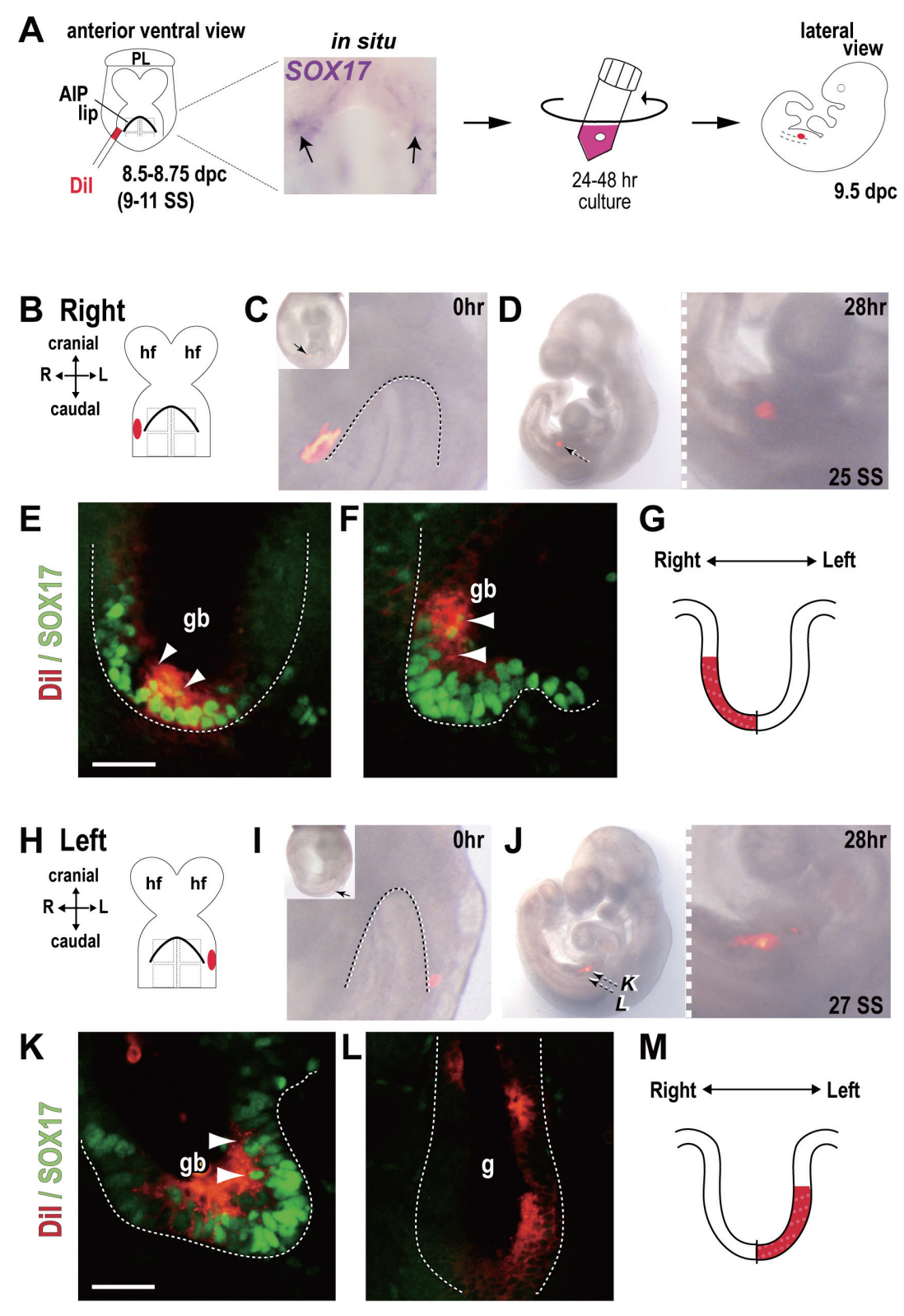

M
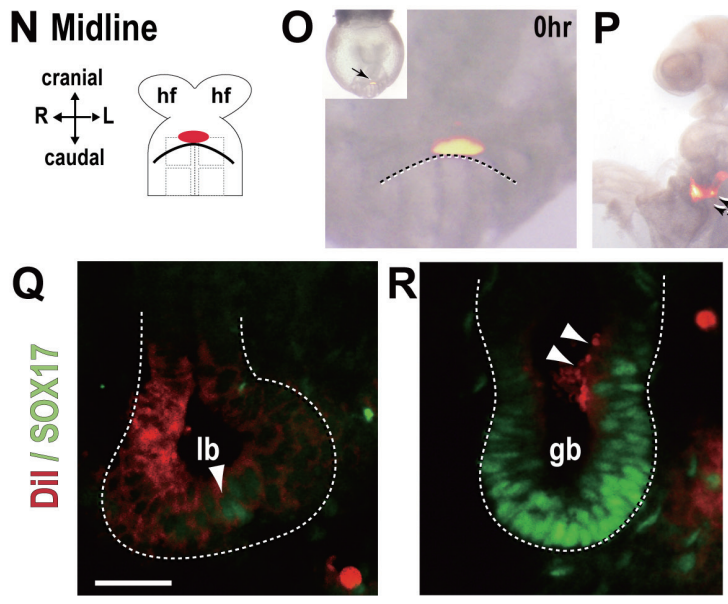

S

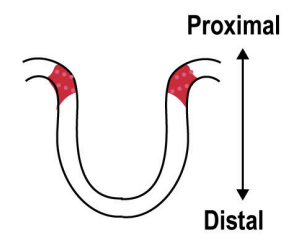


Table 1. Summary of the contribution of DiI-labeled endoderm cells within a small area of the lateral-most domain of the AIP lip (at the first intersomite junction level in 9-11 somite embryos) to the gallbladder primordium after whole embryo cultures for 24-48 $\mathrm{hr}^{\mathrm{a})}$

\begin{tabular}{lccccc}
\hline \multirow{2}{*}{ Marking side } & Total number of & \multicolumn{4}{c}{ Number of embryos with labeled cells in } \\
\cline { 3 - 6 } & embryos analyzed & Gallbladder & Liver bud & Gut $^{\text {b) }}$ & Visceral endoderm \\
\hline Right & 25 & 25 & 1 & 3 & 8 \\
Left & 7 & 3 & 1 & 4 & 4 \\
\hline
\end{tabular}

a) The DiI-labeled cells in the gallbladder primordium were determined by anti-SOX17 immunostaining using serial frozen sections of the cultured embryos. In contrast, the DiI-labeled cells in the liver bud or other regions were histologically estimated. b) Some DiI-labeled cells were found to be located in the posterior gut region including presumptive ventral pancreatic bud.

atic progenitors was reported to express $\operatorname{Sox} 17$ at a certain level before foregut closure [21]. Therefore, it remains unclear whether this defined region within the lateral-most domain of the AIP lip at the first intersomite junction level contains gallbladder progenitors or not.

In general, DiI (fluorescent lipophilic cationic indocarbocyanine dye) fate mapping analysis using whole embryo culture is used to determine cell fate and/or the dynamics of fluorescence-marked cells before and after organ morphogenesis [4, 9]. Several intensive studies have already established detailed fate maps for the liver and pancreas progenitors in early somite-stage embryos before foregut tubulogenesis $[1,4,12,13,23,24]$. However, the location of the gallbladder progenitor remains unclear.

In order to examine the origin of the gallbladder prior to gut closure, we isolated mouse embryos at the 8-11 somite stages (8.5-8.75 dpc) from pregnant wild-type females (ICR strain; SLC, Inc., Hamamatsu, Japan). The animal experiments were performed in accordance with the Guidelines for Animal Use and Experimentation of the University of Tokyo (approval ID: P11-501). The ventral foregut endoderm area of these embryos was labeled with DiI $(0.84 \mu \mathrm{g} / \mu \mathrm{l}, \mathrm{CM}-$ DiI; Life Technologies, Grand Island, NY, U.S.A.) using a micropipette as described previously [5] (Fig. 1A). After rinsing with DMEM, the embryos including the DiI-labeled cells within the appropriate defined position (i.e., the lateralmost domain at the first intersomite junction level) were selected and photographed using a fluorescence microscope to record the DiI-marking position. They were then cultured for $24-48 \mathrm{hr}$ in DMEM supplemented with $75 \%$ rat serum using a rotating-bottle culture system [20]. These cultured embryos were estimated to roughly correspond to the in vivo 9.5-dpc stage (24-30 somite stages [SS]), based on the gross anatomy of the embryonic heart and head (e.g., establishment of left and right ventricles and forebrain formation with enlarging frontonasal process). After culture, these embryos were photographed using a fluorescence microscope. They were then fixed in 4\% PFA-PBS and embedded in OCT compound for serial transverse frozen sectioning (7-10 $\mu \mathrm{m}$ thickness). Finally, in all of the labeled embryos, the location of DiI-positive cells was histologically estimated using immunofluorescence of anti-SOX17 antibodies (a gallbladderspecific marker at $9.5 \mathrm{dpc}$ ) [7].

Whole embryo culture allows for the clear determination of early organogenesis in the foregut endoderm, together with the growth and turning of the entire trunk region, after manipulation with DiI. In all cultured embryos, the majority of DiI-labeled cells were observed to relocate into the internalized gut tube under a fluorescence dissecting microscope (Fig. 1D, 1J, 1P).

In cultured embryos with DiI marking on the right-hand side of the lateral-most domain of the AIP lip (at the level of the first intersomite junction; Fig. 1B and 1C; see also the upper row in Table 1), anti-SOX17 immunostaining using serial frozen sections showed the restricted contribution of DiI-labeled cells to the right ventral (distal) walls of the SOX17-positive gallbladder primordium (Fig. 1D-1G). This clearly suggests that the progenitor population of the gallbladder is present in a small area of the lateral-most domain of the first intersomite-junction level at the 9-11 somite

Fig. 1. Identification of the gallbladder progenitors at the 9-11 somite stages. (A) Schematic representation of DiI fate mapping and whole embryo culture in a rotating bottle using mouse embryos at the $8-11$ somite stages (SS) $(8.5-8.75 \mathrm{dpc})$. The inset photograph shows several Sox17-positive signals (purple staining; arrows) located in the lateral-most domain at the first intersomite junction level of the foregut endoderm by whole mount in situ hybridization (10-somite embryo). (B-S) DiI marking on the right (B-G)- or left (H-M)-side lateral domain at the first intersomite junction level of the foregut endoderm (9-11-somite embryos), in addition to DiI marking at the midline of the AIP lip (N-S) of the foregut endoderm (8-somite embryo). Schematic illustrations (B, G, H, M, N and S) and bright field/fluorescence merged images $(\mathrm{C}, \mathrm{D}, \mathrm{I}, \mathrm{J}$ and $\mathrm{O}, \mathrm{P})$ of the DiI (red fluorescence on the cell surface)-labeled cells before $(0 \mathrm{hr})$ and after whole embryo culture (24-48 hr). Serial transverse sections of the cultured embryos were stained with anti-SOX17 antibody (green fluorescence in the nucleus) at the liver bud (Q), gallbladder (E, F, K and R) and posterior gut (L) levels. After manipulation and 28-hr culture, the DiI-labeled cells (red fluorescence on the apical cell surface) contribute to the gallbladder primordium (white arrowheads in E, F, K, Q and R). Each broken line indicates the outline of the AIP lip (C, I and O) or the gut tube (E, F, K, L, Q and R). In plates C, I and O, each small arrow in the inset (lower-magnified image) indicates the position of DiI-labeled endoderm before culture. The broken arrows in plates D, J and P are the sectioning levels corresponding to plates E, F, K, L and Q, R, respectively. g, gut tube; gb, gallbladder primordium; hf, head fold; lb, liver bud; PL, placental primordium; SS, somite stage; ve, visceral endoderm; vp, ventral pancreas. Bar, $50 \mu \mathrm{m}$. 
stages. Moreover, on the left-hand side of DiI-labeled embryos at the same stages (Fig. 1H and 1I; see the lower row in Table 1), DiI-positive signals were frequently observed in the left ventral (proximal) walls of the gallbladder primordium (Fig. 1J-1K), which confirms the central fusion of both the left and right lateral domains of the foregut endodermal cells at the ventral midline of the gallbladder primordium. Interestingly, some of the DiI-labeled cells on the left-sided lateral region appear to expand into a wider region of the gut tube, resulting in a slender and elongated arrangement of DiI-labeled cells along the gut tube from the gallbladder primordium (see the right inset of Fig. 1J; Fig. 1L). Such left/right asymmetric distribution is also consistent with the asymmetric Sox 17 expression in the lateral-most domains of the AIP lip, in which the Sox17-positive signals on the right side appear to be higher than that on the left side at 10-11 somite stages [25] (see also inset of Fig. 1A). It was previously shown that the left- and right-side cells of the endodermal sheet, including the liver and pancreatic progenitors, contribute asymmetrically to the gut tube on the anterior and posterior sides $[13,24]$. Such asymmetric contribution may be associated with left-right asymmetric morphogenetic events, such as heart looping and axis rotation [4, 13, 24]. The results of the distribution patterns of the DiI-labeled cells at the lateral-most domain of the foregut sheet at the first intersomite levels (9-11-somite-stage embryos) are summarized in Table 1.

Finally, we examined the contribution of the midline of the AIP lip to the gallbladder region (Fig. $1 \mathrm{~N}$ and 1O). The midline of the AIP lip contains multiple progenitors of the dorsal parts of the thyroid, liver and pancreatic buds as determined by previous fate map studies $[1,24]$. In the cultured embryos, we noticed a striated and smeared elongation of DiI-labeled cells along the AP axis of the gut tube (Fig. 1P). Serial sectioning analysis revealed that DiI-labeled cells are co-localized in the anteroproximal part of the SOX17-positive gallbladder region adjacent to the liver bud region in the cultured embryos (Fig. 1Q-1S). Therefore, it is likely that a part of the proximal wall of the gallbladder primordium may be derived from the midline of the AIP lip, similar to their contribution in the other foregut-derived organs [4, 13, 24].

The present study demonstrated the presence of distinct populations of gallbladder progenitor cells in the lateral-most domains of the ventral foregut endoderm on both the left and right sides, in addition to the midline of the AIP lip before foregut tubulogenesis. Each population appears to contribute to distinct parts of the gallbladder primordium: the left distal, right distal or proximal part of the gallbladder primordium in the 9.5-dpc embryos. At the 9-11 somite stages, the liver progenitors are located within the midline of the AIP lip, anteriorly adjacent to the ventral pancreas progenitors [24]. Recent fate maps have shown that ventral pancreatic progenitors are located in the lateral endoderm in the early somite stages [1] and then in the caudal midline of the AIP lip in the 9-11 somite stages [13], although these fate maps showed slightly different patterns, possibly due to the different genetic backgrounds used in each experiment. Compared to these two positions for the liver and pancreatic progeni- tors in the midline of the AIP lip, the gallbladder progenitors at the 9-11 somite stages appear to be restricted to a defined area lateral-most to the midline position of both the liver and pancreatic progenitors. Since several endoderm cells begin to express Sox17 in the lateral-most region underling the mesenchymal layers, including the vitelline veins of the yolk primordium [25], this defined region is likely the initial specification site of the gallbladder primordium and these underlining tissues may be a potential inductive signaling source for gallbladder specification in early somitogenesis.

In conclusion, this fate-map study provides direct evidence showing that the bulk of the gallbladder progenitors are located in the lateral-most domains at the first intersomite level of the ventral foregut endoderm at 8.5-8.75 dpc (9-11 somite stages). Such information should be helpful not only for further analysis of the inductive signals of gallbladder progenitors but also for the upstream analysis of Sox 17 reactivation in early organogenesis in the foregut endoderm of mammals.

ACKNOWLEDGMENTS. The authors wish to deeply thank Prof. Yukio Saijoh (Department of Neurobiology and Anatomy, The University of Utah, U.S.A.) for his kind and valuable suggestions on the DiI fate-mapping analyses. The authors also wish to thank Dr. Miyuri Kawasumi, Dr. Yoshiko Kuroda, Dr. Hiroki Higashiyama, Dr. Hiroyuki Sumitomo and Ms. Yuki Uchiyama for their helpful support, and Ms. Itsuko Yagihashi for her secretarial assistance. This work was supported by financial grants from the Ministry of Education, Science, Sports and Culture of Japan.

\section{REFERENCES}

1. Angelo, J. R., Guerrero-Zayas, M. I. and Tremblay, K. D. 2012. A fate map of the murine pancreas buds reveals a multipotent ventral foregut organ progenitor. PLOS ONE 7: e40707. [Medline] [CrossRef]

2. Arterbery, A. S. and Bogue, C. W. 2014. Endodermal and mesenchymal cross talk: a crossroad for the maturation of foregut organs. Pediatr. Res. 75: 120-126. [Medline] [CrossRef]

3. Engert, S., Liao, W. P., Burtscher, I. and Lickert, H. 2009. Sox172A-iCre: a knock-in mouse line expressing Cre recombinase in endoderm and vascular endothelial cells. Genesis 47: 603-610. [Medline] [CrossRef]

4. Franklin, V., Khoo, P. L., Bildsoe, H., Wong, N., Lewis, S. and Tam, P. P. 2008. Regionalisation of the endoderm progenitors and morphogenesis of the gut portals of the mouse embryo. Mech. Dev. 125: 587-600. [Medline] [CrossRef]

5. Hara, K., Kanai-Azuma, M., Uemura, M., Shitara, H., Taya, C., Yonekawa, H., Kawakami, H., Tsunekawa, N., Kurohmaru, M. and Kanai, Y. 2009. Evidence for crucial role of hindgut expansion in directing proper migration of primordial germ cells in mouse early embryogenesis. Dev. Biol. 330: 427-439. [Medline] [CrossRef]

6. Kanai-Azuma, M., Kanai, Y., Gad, J. M., Tajima, Y., Taya, C., Kurohmaru, M., Sanai, Y., Yonekawa, H., Yazaki, K., Tam, P. P. and Hayashi, Y. 2002. Depletion of definitive gut endoderm in Sox17null mutant mice. Development 129: 2367-2379. [Medline]

7. Kanai, Y., Kanai-Azuma, M., Noce, T., Saido, T. C., Shiroishi, T., Hayashi, Y. and Yazaki, K. 1996. Identification of two Sox 17 
messenger RNA isoforms, with and without the high mobility group box region, and their differential expression in mouse spermatogenesis. J. Cell Biol. 133: 667-681. [Medline] [CrossRef]

8. Kim, I., Saunders, T. L. and Morrison, S. J. 2007. Sox17 dependence distinguishes the transcriptional regulation of fetal from adult hematopoietic stem cells. Cell 130: 470-483. [Medline] [CrossRef]

9. Kinder, S. J., Tan, S. S. and Tam, P. P. 2000. Cell grafting and fate mapping of the early-somite-stage mouse embryo. Methods Mol. Biol. 135: 425-437. [Medline]

10. Lawson, K. A. and Pedersen, R. A. 1987. Cell fate, morphogenetic movement and population kinetics of embryonic endoderm at the time of germ layer formation in the mouse. Development 101: 627-652. [Medline]

11. Matsui, T., Kanai-Azuma, M., Hara, K., Matoba, S., Hiramatsu, R., Kawakami, H., Kurohmaru, M., Koopman, P. and Kanai, Y. 2006. Redundant roles of Sox 17 and Sox 18 in postnatal angiogenesis in mice. J. Cell Sci. 119: 3513-3526. [Medline] [CrossRef]

12. Matsuura, K., Katsumoto, K., Fukuda, K., Kume, K. and Kume, S. 2009. Conserved origin of the ventral pancreas in chicken. Mech. Dev. 126: 817-827. [Medline] [CrossRef]

13. Miki, R., Yoshida, T., Murata, K., Oki, S., Kume, K. and Kume, S. 2012. Fate maps of ventral and dorsal pancreatic progenitor cells in early somite stage mouse embryos. Mech. Dev. 128: 597-609. [Medline] [CrossRef]

14. Nobuhisa, I., Osawa, M., Uemura, M., Kishikawa, Y., Anani, M., Harada, K., Takagi, H., Saito, K., Kanai-Azuma, M., Kanai, Y., Iwama, A. and Taga, T. 2014. Sox17-mediated maintenance of fetal intra-aortic hematopoietic cell clusters. Mol. Cell. Biol. 34: 1976-1990. [Medline] [CrossRef]

15. Pfister, S., Jones, V. J., Power, M., Truisi, G. L., Khoo, P. L., Steiner, K. A., Kanai-Azuma, M., Kanai, Y., Tam, P. P. and Loebel, D. A. 2011. Sox 17-dependent gene expression and early heart and gut development in Sox17-deficient mouse embryos. Int. J. Dev. Biol. 55: 45-58. [Medline] [CrossRef]

16. Saito, Y., Kojima, T. and Takahashi, N. 2013. The septum transversum mesenchyme induces gall bladder development. Biol. Open 2: 779-788. [Medline] [CrossRef]

17. Sakamoto, Y., Hara, K., Kanai-Azuma, M., Matsui, T., Miura, Y., Tsunekawa, N., Kurohmaru, M., Saijoh, Y., Koopman, P. and Kanai, Y. 2007. Redundant roles of Sox 17 and Sox 18 in early cardiovascular development of mouse embryos. Biochem. Biophys. Res. Commun. 360: 539-544. [Medline] [CrossRef]

18. Saund, R. S., Kanai-Azuma, M., Kanai, Y., Kim, I., Lucero, M.
T. and Saijoh, Y. 2012. Gut endoderm is involved in the transfer of left-right asymmetry from the node to the lateral plate mesoderm in the mouse embryo. Development 139: 2426-2435. [Medline] [CrossRef]

19. Shimoda, M., Kanai-Azuma, M., Hara, K., Miyazaki, S., Kanai, Y., Monden, M. and Miyazaki, J. 2007. Sox17 plays a substantial role in late-stage differentiation of the extraembryonic endoderm in vitro. J. Cell Sci. 120: 3859-3869. [Medline] [CrossRef]

20. Sturm, K. and Tam, P. P. 1993. Isolation and culture of whole postimplantation embryos and germ layer derivatives. Methods Enzymol. 225: 164-190. [Medline] [CrossRef]

21. Spence, J. R., Lange, A. W., Lin, S. C., Kaestner, K. H., Lowy, A. M., Kim, I., Whitsett, J. A. and Wells, J. M. 2009. Sox17 regulates organ lineage segregation of ventral foregut progenitor cells. Dev. Cell 17: 62-74. [Medline] [CrossRef]

22. Tam, P. P., Kanai-Azuma, M. and Kanai, Y. 2003. Early endoderm development in vertebrates: lineage differentiation and morphogenetic function. Curr. Opin. Genet. Dev. 13: 393-400. [Medline] [CrossRef]

23. Tam, P. P., Khoo, P. L., Lewis, S. L., Bildsoe, H., Wong, N., Tsang, T. E., Gad, J. M. and Robb, L. 2007. Sequential allocation and global pattern of movement of the definitive endoderm in the mouse embryo during gastrulation. Development 134: 251-260. [Medline] [CrossRef]

24. Tremblay, K. D. and Zaret, K. S. 2005. Distinct populations of endoderm cells converge to generate the embryonic liver bud and ventral foregut tissues. Dev. Biol. 280: 87-99. [Medline] [CrossRef]

25. Uemura, M., Hara, K., Shitara, H., Ishii, R., Tsunekawa, N., Miura, Y., Kurohmaru, M., Taya, C., Yonekawa, H., Kanai-Azuma, M. and Kanai, Y. 2010. Expression and function of mouse Sox 17 gene in the specification of gallbladder/bile-duct progenitors during early foregut morphogenesis. Biochem. Biophys. Res. Commun. 391: 357-363. [Medline] [CrossRef]

26. Uemura, M., Ozawa, A., Nagata, T., Kurasawa, K., Tsunekawa, N., Nobuhisa, I., Taga, T., Hara, K., Kudo, A., Kawakami, H., Saijoh, Y., Kurohmaru, M., Kanai-Azuma, M. and Kanai, Y. 2013. Sox17 haploinsufficiency results in perinatal biliary atresia and hepatitis in C57BL/6 background mice. Development 140: 639-648. [Medline] [CrossRef]

27. Zaret, K. S. 2008. Genetic programming of liver and pancreas progenitors: lessons for stem-cell differentiation. Nat. Rev. Genet. 9: 329-340. [Medline] [CrossRef]

28. Zorn, A. M. and Wells, J. M. 2009. Vertebrate endoderm development and organ formation. Annu. Rev. Cell Dev. Biol. 25: 221-251. [Medline] [CrossRef] 